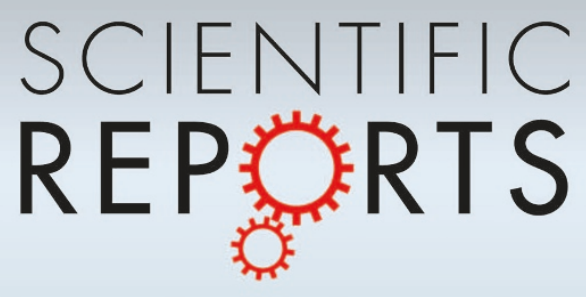

OPEN

SUBJECT AREAS:

LIVER DISEASES

EXPERIMENTAL MODELS OF

DISEASE

Received

10 June 2014

Accepted

8 September 2014

Published

29 September 2014

Correspondence and requests for materials should be addressed to L.W. (weilixin_smmu@ 163.com)

\section{Cell-based therapy for acute and chronic liver failures: Distinct diseases, different choices}

\author{
Kai Sun' ${ }^{1}$ Xuqin $\mathrm{Xie}^{2,3}$, Jing Xie ${ }^{4}$, Shufan Jiao', Xiaojing Chen' ${ }^{1}$ Xue Zhao', Xin Wang ${ }^{5,6,7}$ \& Lixin Wei ${ }^{1,2}$
}

\begin{abstract}
${ }^{1}$ Central laboratory, Ren Ji Hospital, School of Medicine, Shanghai Jiao Tong University, Shanghai, China, ${ }^{2}$ Tumor Immunology and Gene Therapy Center, Eastern Hepatobiliary Surgery Hospital, The Second Military Medical University, Shanghai, China,

${ }^{3}$ Department of Medical Biotechnology and Translational Medicine, University of Milan, Milan, Italy, ${ }^{4}$ Department of Gynecology, Shanghai Tianshan Hospital, Shanghai, China, ${ }^{5}$ The Key Laboratory of National Education Ministry for Mammalian Reproductive Biology and Biotechnology, Inner Mongolia University, Huhhot, P.R.China, ${ }^{6}$ Department of Laboratory Medicine and Pathology, University of Minnesota, Minneapolis, Minnesota, MN, USA, ' Hepatoscience Incorporation, Palo Alto, CA.
\end{abstract}

Cell-based therapies (CBTs) are considered the effective approaches to treat liver failure. However, which cell type is the most suitable source of CBTs for acute liver failure (ALF) or chronic liver failure (CLF) remains unclear. To investigate this, mature hepatocytes in adult liver (adult HCs), fetal liver cells (FLCs), induced hepatic stem cells (iHepSCs) and bone marrow derived mesenchymal stromal cells (BMSCs) were used to CBTs for ConA-induced ALF and Fah-deficient induced CLF in mice. The results showed that only BMSCs remitted liver damage and rescued ALF in ConA-treated mice. In this process, BMSCs inhibited ConA-induced inflammatory response by decreasing the mRNA expressions of TNF- $\alpha$, IFN- $\gamma$ and FasL and increasing IL-10 mRNA expression. However, in the CLF model, not BMSCs but adult HCs transplantation lessened liver injury, recovered liver function and rescued the life of Fah-/- mice after NTBC withdrawal. Further study showed that adult HCs offered more effective liver regeneration compared to other cells in Fah-/- mice without NTBC. These results demonstrated that BMSCs and adult HCs are the optimal sources of CBTs for ConA-induced ALF and Fah-deficient induced CLF in mice, respectively. This finding deepens our understanding about how to select a proper CBT for different liver failure.

iver failure, the critical deterioration of liver function, is a complex fatal liver disease. It has a variety of etiologies, including viral hepatitis, excessive alcohol, drug-induced hepatotoxicity, metabolic liver disease, and uncertain causes ${ }^{1}$. Depending on the duration of the disease, liver failure can be roughly distinguished into acute liver failure (ALF) and chronic liver failure (CLF).

ALF, a rare but sudden clinical syndrome in people without pre-existing serious liver disease, results from fast and extensive hepatic necrosis and causes severe jaundice, coagulopathy, hepatic encephalopathy, and even multi-organ failure ${ }^{2}$. CLF often occurs in patients with incurable chronic liver disease or stable cirrhosis and usually is accompanied by continuous inflammation. CLF leads to metabolic disorders of various toxins and presents irreversible chronic loss of liver function ${ }^{3}$. Additionally, a sudden event such as severe infections, sepsis and excessive alcohol intake can cause dramatic damage of hepatic tissue in patients with chronic liver disease, which is termed as acute-on-chronic liver failure ${ }^{4}$.

Although modern medical treatment has continuous improvement, orthotopic liver transplantation (OLT) is still considered the most effective therapy in the patients suffering from life-threatening liver failure. However, shortage of donor liver, contraindications and expensive charge greatly limits its widespread use. Cell-based therapies (CBTs) are deemed to the suitable alternative therapeutic approaches for liver failure owing to its fewer traumas, less cost, relatively simple technique, reversibility and repeatability. Transplanted cells could remit the deterioration of liver function and promote liver regeneration, which might avoid OLT entirely or at least be a bridge to $\mathrm{OLT}^{5}$. Several laboratorial and clinical studies showed that various cells, including mature hepatocytes in adult liver (adult HCs), fetal liver cells (FLCs), hepatic stem/progenitor cells (HSPCs), mesenchymal stromal cells (MSCs), are the potential sources of CBTs for liver failure ${ }^{6,7}$.

In the present study, we attempt to explore the differences on the therapeutic effects of these cells in treatments of ALF and CLF. We isolated adults HCs and bone marrow derived MSCs (BMSCs) from adult C57BL/6 mice and FLCs from the liver of C57BL/6 mice embryos at day 13.5. Induced hepatic stem cells (iHepSCs) from MEFs, 
which has bi-directional differentiation potential in vitro and in vivo, are used as HSPCs ${ }^{8}$. On the other hand, we utilized Concanavalin A (ConA) induced acute liver injury to mimic ALF and fumarylacetoacetate hydrolase-deficient (Fah-/-) induced chronic liver injury to mimic CLF. ConA, a plant lectin and $\mathrm{T}$ cell mitogen, is widely used to induce rapid, severe and dose-dependent hepatitis and subsequent liver injury in mice model ${ }^{9}$. Fah is a metabolic enzyme that catalyzes the last step of tyrosine catabolism. Fah deficiency causes the accumulation of two tyrosine metabolites, maleylacetoacetate and fumarylacetoacetate, which are responsible for liver damage by leading to hepatocytes necrosis. 2-(2-nitro-4-trifluoromethylbenzoyl) -1,3cyclohexanedione (NTBC) can decrease the accumulation of maleylacetoacetate and fumarylacetoacetate by inhibiting hydroxyphenylpyruvate, the second enzyme of tyrosine metabolic pathways. Fah-/mice require NTBC for maintaining liver function and survival. If NTBC treatment is withdrawn, Fah-/- mice undergo CLF and subsequent death ${ }^{10,11}$. Then we used these two kinds of mice models to investigate the most appropriate candidate of CBTs among these four kinds of cells in the ALF and CLF treatments.

\section{Results}

Only BMSCs transplantation can rescue ConA-induced ALF in mice. Firstly, we used immunofluorescent staining to identify phenotypic characterizations of adult HCs, FLCs and iHepSCs that would be utilized in our experiments. The results showed that all of adult HCs, FLCs and iHepSCs expressed albumin (ALB), which is a classic hepatocyte lineage marker and is widely used to indicate liver function. And none of adult HCs, a small population of FLCs and all of iHepSCs were bipotent cells, which co-expressed $\alpha$-fetoprotein (AFP) and cytokeratin (CK) -19 (Figure 1a). Meanwhile, flow cytometry analysis revealed that BMSCs expressed specific surface markers, including CD29, CD90 and CD105, and were negative for CD14, monocyte surface marker, and CD34 and CD45, two hematopoietic cell-surface markers (Figure 1b). And BMSCs exhibited the ability of differentiating into adipocytes and osteoblast-like cells (Figure 1c). Therefore, the cell used in our experiments accorded with their corresponding characterizations.

Then the mice were separately received intrasplenical injection with adult HCs, FLCs, iHepSCs, BMSCs and the same volume of saline solution 2 hours after ConA injection $(25 \mathrm{mg} / \mathrm{kg})$. The cohort of mice monitored for survival demonstrated that survival percentage of ConA + Saline group was just 12.5\% (1/8) 19 hours after ConA injection. The ConA + BMSCs treated mice exhibited longer mean survival time compared to that of ConA + Saline treated mice. However, adult HCs, FLCs and iHepSCs transplantations had no significant influence on the survival times of ConA-treated mice (Figure 2). These results showed that only BMSCs transplantation rescued ConA-induced ALF in mice.

Transplanted BMSCs lessens ConA-induced liver injury by reducing inflammatory responses. To investigate why only BMSCs transplantation can remit ConA-induced ALF, we explored the changes causing by cell transplantation in the ConA-treated mice. Firstly, we sacrificed the mice 10 hours after ConA injection, and then examined liver pathological structures and the serum markers of liver injury and functions. Hematoxylin and eosin (H\&E) staining revealed that ConA caused prominent lymphocyte infiltration, the obvious accumulation of red blood cell in hepatic sinusoid, which hints the serious injury of vascular endothelial cell in the liver, and severe hepatocyte necrosis. BMSCs transplantation reduced the ConA-induced damages to liver cells and vascular endothelial cells and lymphocyte infiltration, but adult HCs, FLCs and iHepSCs transplantations had no remarkable influence on ConA-induced liver injury (Figure 3a). Further serum examination also showed that ConA resulted in the increases of the levels of alanine aminotransferase (ALT) and aspartate aminotransferase (AST), two classical markers of liver injury, and total bilirubin (Tbil), an indicator of impaired liver function, and the decrease of ALB level. Adult HCs, FLCs and iHepSCs transplantations still had no significant impact on the ConA-induced changes of serum markers, but BMSCs transplantation retarded liver damage and improved liver function in the ConA-treated mice (Figure 3b-e). These results indicated that only BMSCs transplantation decreases ConA-induced acute liver injury in mice.

The increases of TNF- $\alpha$, IFN- $\gamma$, IL-2, IL-4, IL-5 and FasL, all of which are mainly produced by lymphocytes and kupffer cells, directly contribute to ConA-induced severe immune-mediated hepatitis in mice ${ }^{9,12-16}$. IL-10 is a crucial factor in the tolerance of ConA-induced hepatitis ${ }^{17}$. We detected the influence of cell transplantations on these cellular factors. The results indicated that BMSCs transplantation inhibited TNF- $\alpha$, IFN- $\gamma$ and FasL mRNA expressions in the lymphocytes and kupffer cells of ConA-treated liver, and increased IL-10 mRNA expression in ConA-treated liver. However, there were no obvious differences on the mRNA expressions of TNF- $\alpha$, IFN- $\gamma$, FasL and IL-10 in the ConA-treated mice liver due to adult HCs, FLCs or iHepSCs transplantation (Figure 4). Meanwhile, all kinds of cell transplantation had no significant impact on IL-2, IL-4 and IL-5 mRNA expressions in the lymphocytes and kupffer cells of ConA-treated liver (Supplementary Fig. S1 online). These results suggested that BMSCs transplantation restrained ConA-induced acute liver injury through decreasing TNF- $\alpha$, IFN- $\gamma$ and FasL expressions and increasing IL-10 expression.

Adult HCs is the optimal donor cells for treating CLF. Here, we used Fah-/- mice to explore the impacts of these four kinds of CBTs on CLF. We injected intrasplenically adult HCs, FLCs, iHepSCs, BMSCs and the same volume of saline solution into Fah -/- mice without NTBC, respectively. After 25 days from cell injections, only adult HCs transplantation prominently remitted the decrease of mice weight arising from NTBC withdrawal (Figure 5a). The cohort of mice monitored for survival also demonstrated that all Fah-/- mice of NTBC(-) + Saline group died in 37 days. And only NTBC(-) + adult HCs group showed longer mean survival time than NTBC(-) + Saline group. FLCs, iHepSCs and BMSCs transplantations did not significantly prolong the survival time of Fah-/- mice without NTBC (Figure 5b). These results suggested that adult HCs was the most suitable donor cells for treating Fah-deficient induced CLF compared with FLCs, iHepSCs and BMSCs.

Adult HCs remits CLF by supplying efficient liver regeneration. Then we further to investigate why adult HCs transplantation is the most effective CBT for Fah-deficient induced CLF. Fah-/- mice were sacrificed 25 days after cell injections. Serum examination exhibited that NTBC withdrawal caused the increase of ALT, AST and Tbil levels, and the reduction of ALB level in the Fah-/- mice. Adult HCs transplantation prominently inhibited Fah-deficient induced liver injury and improved liver function. However, FLCs, iHepSCs and BMSCs transplantations did not lead to conspicuous changes in liver serum markers in the Fah-/- mice without NTBC (Figure 6a-d). Toxicity of tyrosine metabolic intermediate is the main reason causing liver cell injury in Fah-/- mice. The decrease of liver damage and the recovery of liver function implied that transplanted adult HCs had already successful regeneration and exerted the function of tyrosine metabolism. Fah-/- mice model can be used to evaluate the regeneration of exogenous liver cells through immunohistochemical staining of Fah. Therefore, we further analyzed liver samples from these recipients. The results revealed that Fah-/- mice with adult HCs transplantation had a higher percentage of Fah-positive cells in the total hepatocytes than that of the Fah-/- mice with FLCs, iHepSCs or BMSCs transplantation (Figure 6e-f). These results demonstrated that adult HCs supplied the most efficient hepatocyte regeneration compared to FLCs, iHepSCs and BMSCs, and finally rescued Fah-deficient induced CLF. 

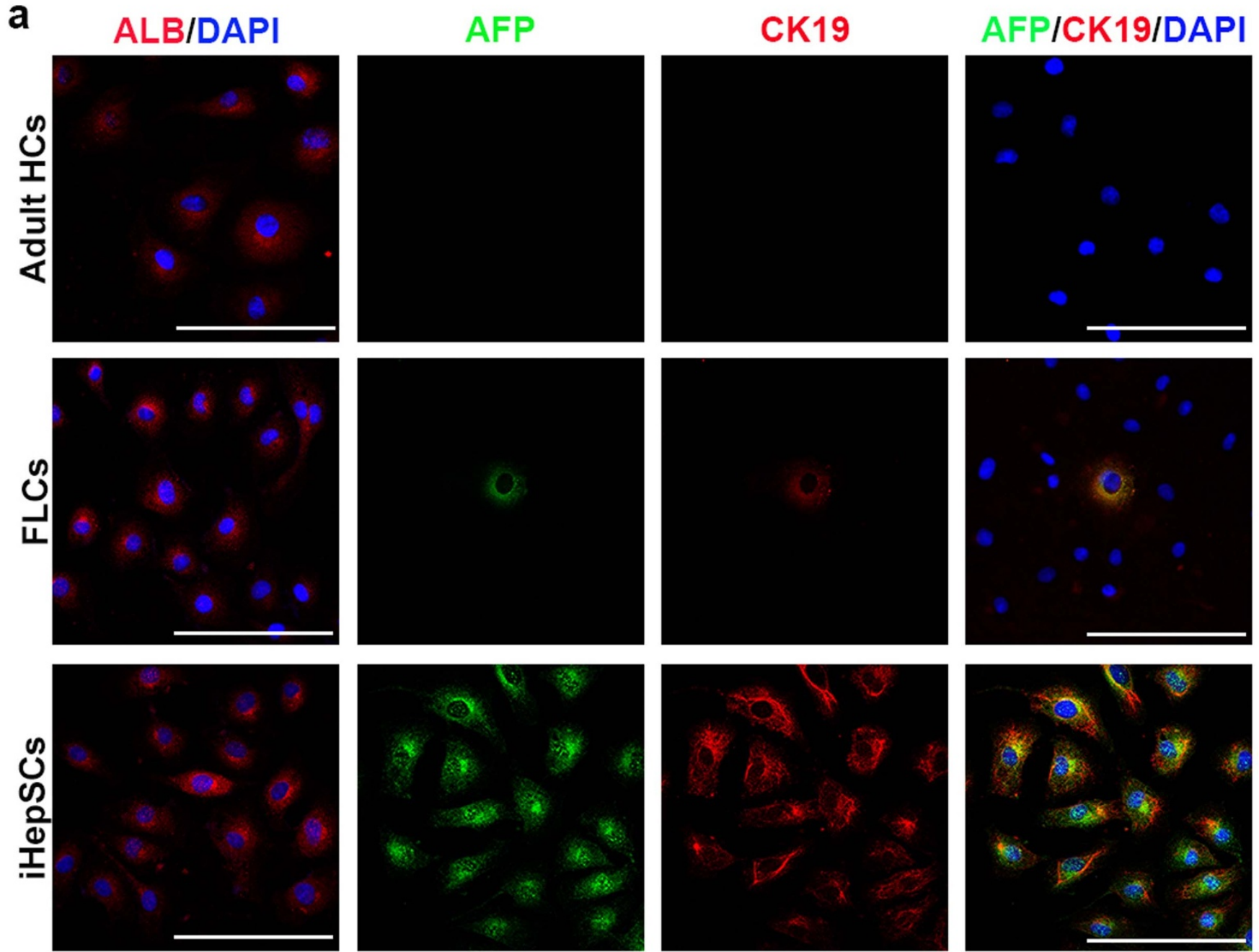

b
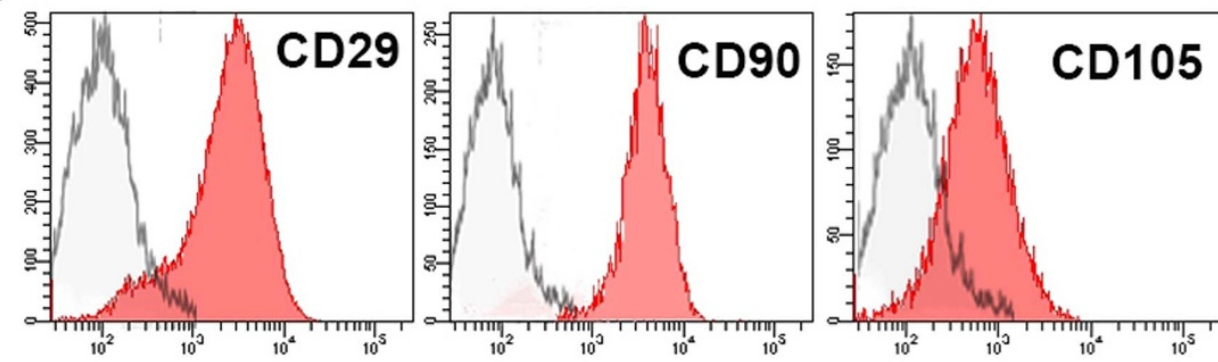

C
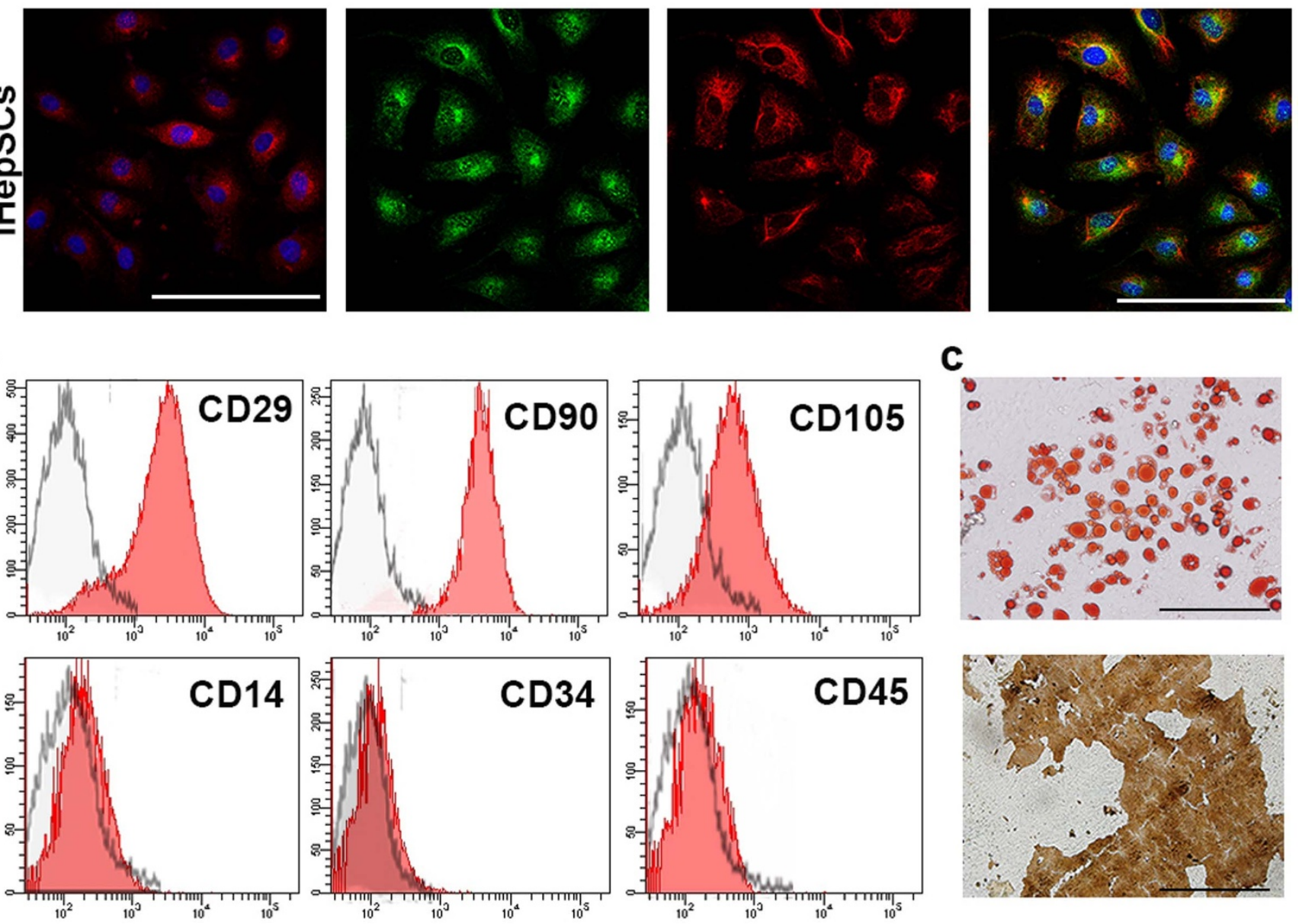

Figure 1 Identification of adult HCs, FLCs, iHepSCs and BMSCs. (a) Adult HCs, FLCs and iHepSCs were detected by immunofluorescence staining for expressions of ALB, AFP and CK19. Bar: $100 \mu \mathrm{m}$. (b) Flow cytometry analysis showed that BMSCs expressed CD29, CD90 and CD105 and were negative for CD14, CD34, and CD45. (c) BMSCs were induced to adipogenic (upper) and osteogenic (lower) differentiation. The cells were treated with oil red O staining and von Kossa staining, respectively. Magnification $\times 200$. Bar: $100 \mu \mathrm{m}$. 


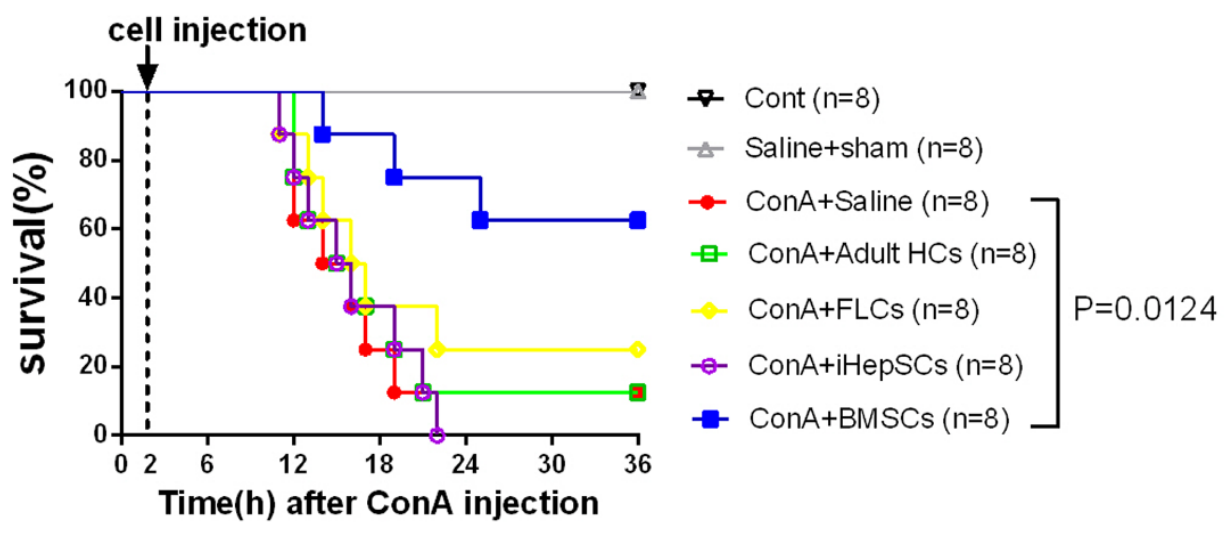

Figure $2 \mid$ The survival times of ConA-treated mice with CBTs. Survival curves of the indicated groups. Log rank test revealed the difference between the curves of ConA + Saline and ConA + BMSCs groups $(n=8 ; P=0.0124)$.

\section{Discussion}

In this study, we evaluated the treatment efficiency of four kinds of CBTs for ALF and CLF, respectively. Our finding demonstrated that the most appropriate CBT is different between ALF and CLF treatments. In the CBT for ALF, BMSCs transplantation is the optimal candidate, which can timely and efficaciously reduce drastic inflammatory response and thereby decreases severe liver injury and rescues liver failure. However, in the CBT for CLF, adult HCs is the primary choice. Compared with FLCs, iHepSCs and BMSCs, adult $\mathrm{HCs}$ can provide the quickest liver regeneration and thereby effectively restore liver function.

CBT is a promising alternative treatment for patients with endstage liver disease, especially who cannot wait or afford to undergo OLT. Many kinds of cells are deemed to the potential sources of CBT. As early as 1976, adult HCs transplantation was used to treat an inborn error of liver metabolism in rodent model ${ }^{18}$. Since then, many delightful results about the effects of adult HCs transplantation for liver failure were observed in different animal models ${ }^{19}$. Although adult HCs transplantation has numerous advantages compared to OLT, it still is subject to the limited source in the clinical application. Besides induced hepatocyte-like cells ${ }^{20,21}$, more cell sources are also considered in the application of CBT for liver failure. HSPCs is able to differentiate into mature hepatocyte, and was observed its successful liver repopulation in several animal models $\mathrm{s}^{22-24}$. The low number of HSPCs in adult liver and the difficulty of large-scale expansion in vitro of HSPCs limited the application of HSPCs transplantation in the past, but iHepSCs supports a novel method to overcome these difficulties $^{8}$. FLCs, which contains some HSPCs, some immature hepatocytes and many mature hepatocytes, is also confirmed to have ability of liver regeneration in normal or damaged liver ${ }^{25}$. MSCs is another source of HSPCs, which has been isolated from various tissues, such as bone marrow, adipose tissue and so on. Some studies have demonstrated that MSCs can differentiate into hepatocyte under defined conditions in vitro ${ }^{26}$, and regenerate the liver in vivo ${ }^{27}$. a
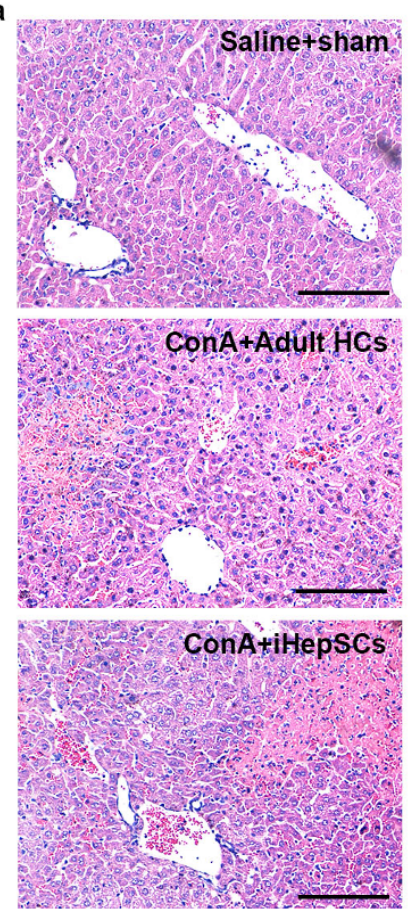
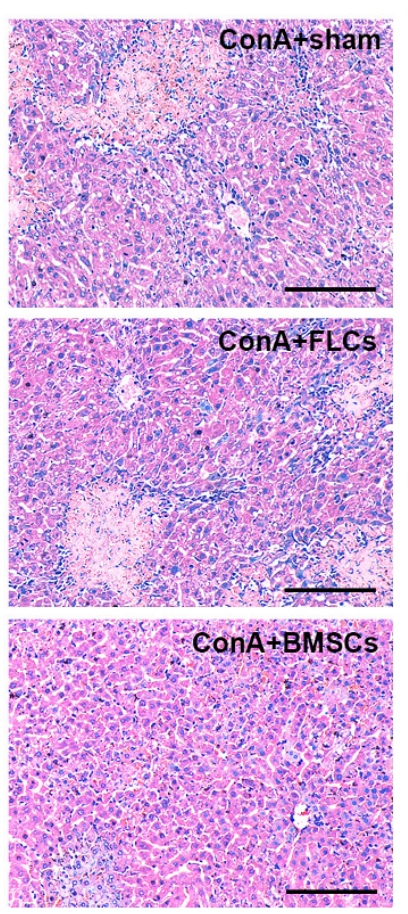

b

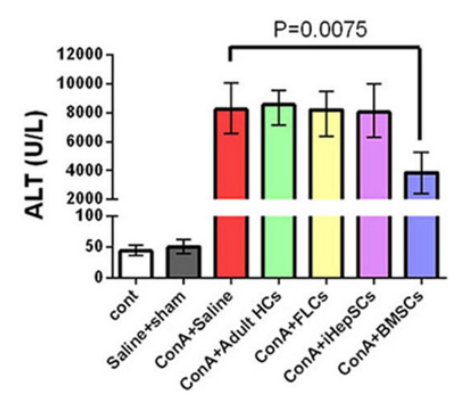

d

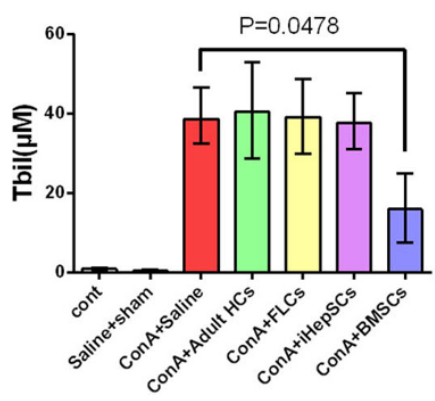

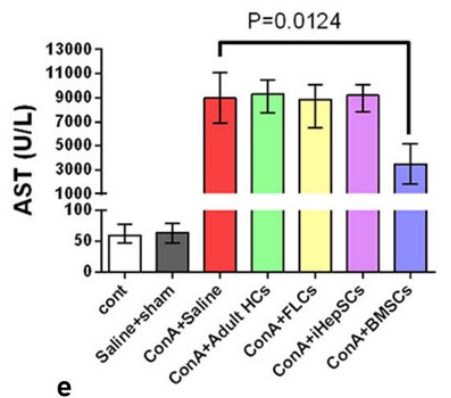

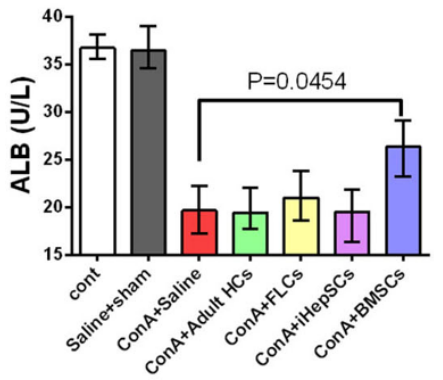

Figure 3 The liver injury of ConA-treated mice with CBTs. (a-e) The mice of the indicated groups were sacrificed 10 hours after ConA injection. (A) Mice liver sections of indicated groups were stained with H\&E. Representative images were shown with an original magnification $\times 400$. Bar: $100 \mu$ m. (b-e) The serum levels of ALT(b), AST(c), Tbil(d) and ALB(e) of mice from the indicated groups were detected. Data were represented as mean with range $(\mathrm{n}=5)$. 
a

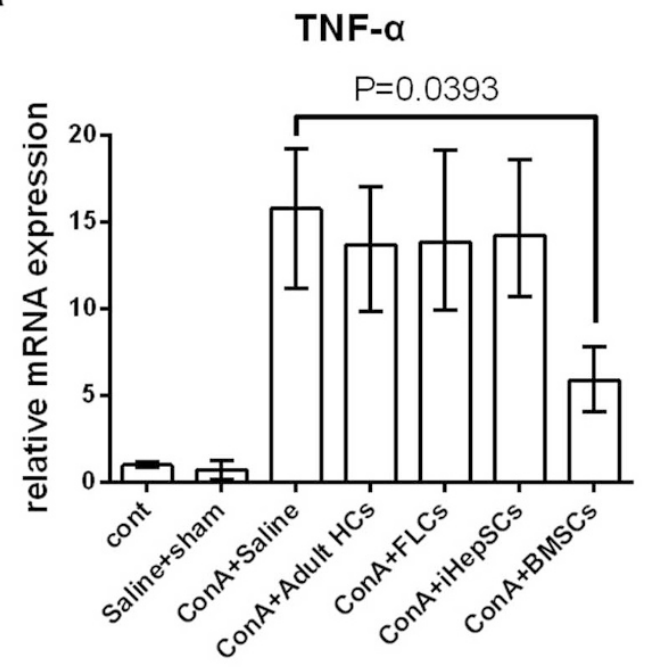

C

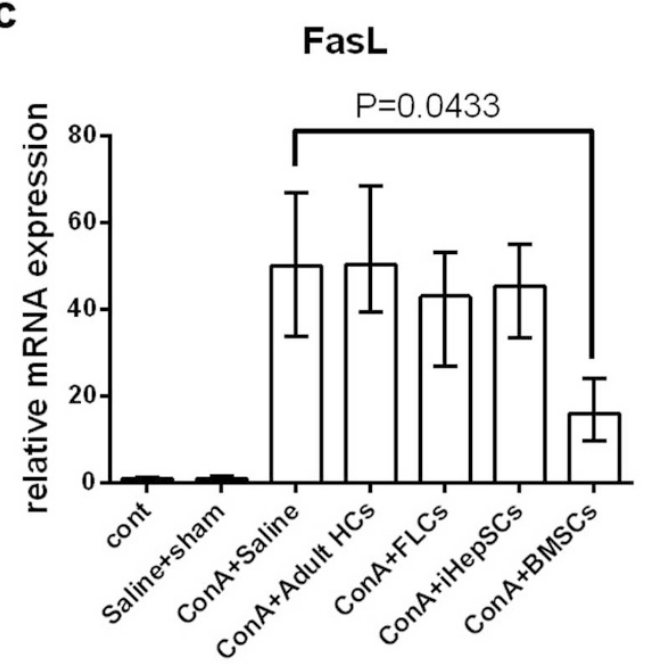

b

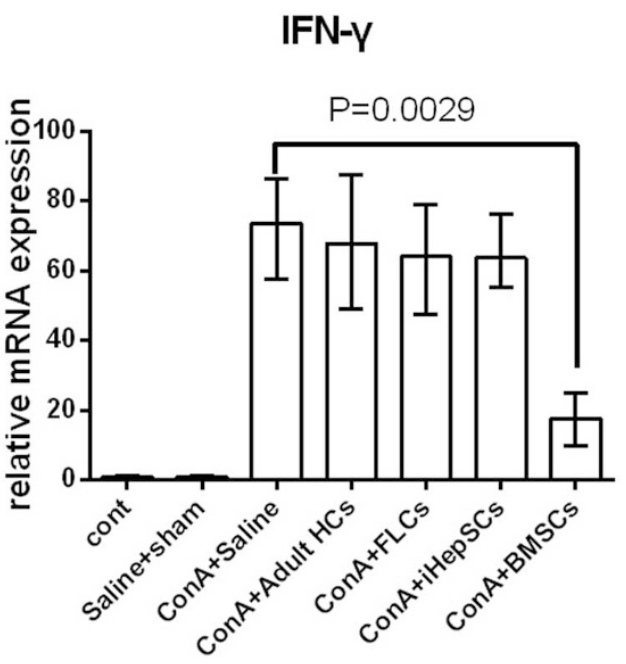

d

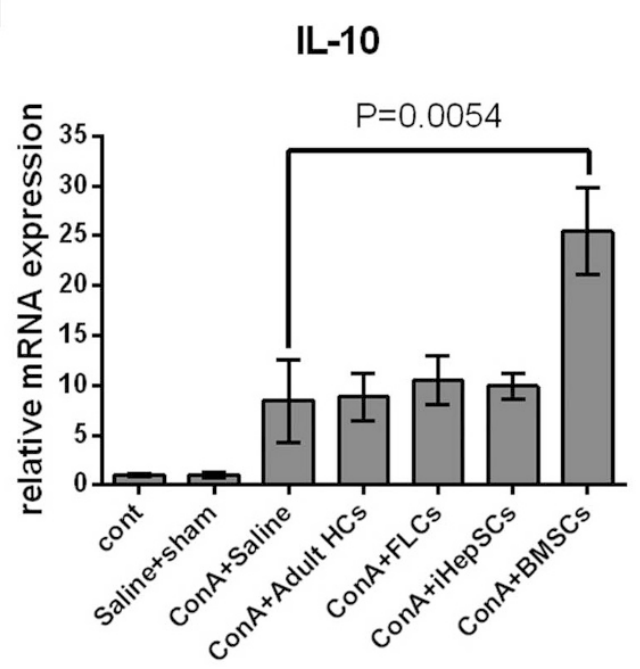

Figure 4 The mRNA expressions of inflammation-associated factors in the livers of ConA-treated mice with CBTs. (a-d) Mice were treated as

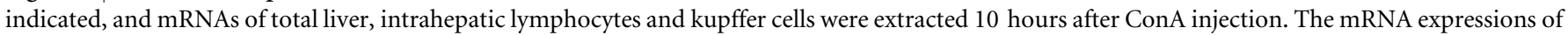

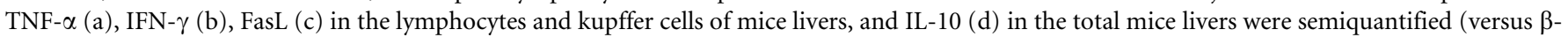
actin). Data were represented as mean with range $(\mathrm{n}=5)$.

Although all these cells have the potential to treat liver failure, the differences among them in the treatment of liver failure are worth to explore.

Fulminant hepatitis is one of the main causes of ALF. T-cell-rich infiltration and subsequent immune response play the principal role in hepatocyte destruction in this kind of disease ${ }^{28}$. In its mimetic mice model, high dose ConA-induced liver injury, except for BMSCs, three other kinds of cells cannot rescue the mice with ALF. Moreover, the mechanism of the rescue of BMSCs to ALF is involved in the inhibition of inflammatory response resulting in liver injury but not the promotion of liver regeneration. We were not observed Fah-positive hepatocytes derived from transplanted BMSCs in the Fah-/- mice with NTBC after ConA injection. Cao H, et al. reported ${ }^{29}$ that human placental MSCs rescued D-galactosamine induced pig ALF and were the source of hepatocyte-like cells at 5 months. However, from the beginning of their ALF model, MSCs had already exerted their therapeutic effect. Immunosuppression ${ }^{30,31}$ may be the fundamental function of MSCs in their model. Salomone F, et al. reported $^{32}$ that adipose tissue-MSCs is effective in treating acetaminophen induced acute liver injury by inhibiting liver stress and inflammatory signaling. We also found that BMSCs transplantation resulted in the decrease of TNF- $\alpha$, IFN- $\gamma$ and FasL expressions and the increase of IL-10 expression in the ConA-treated mice liver. Therefore, BMSCs transplantation can effectively remit immuneinvolved ALF, but its exact mechanism still requires further investigation. Previous studies showed that transplanted hepatocytes in $\mathrm{N}$-dimethylonitrosamine or D-galactosamine induced ALF had remarkable therapeutic effect ${ }^{33,34}$. However, adult HCs, FLCs and iHepSCs transplantations have no significant impacts on the ConA-induced liver injury. That the course of ALF is too short for the cells to regenerate efficiently and the inflammatory response in ConA-induced liver injury is too fierce for the cells to survive may be two possible reasons resulting in this phenomenon.

Different from ALF, CLF has not dramatic but persistent and relatively mild liver injury. Transplanted cells have abundant time to engraft into the injured liver and trigger the process of liver regeneration in CLF model. For having a clear contrast in the efficiency of liver regeneration among these cells, we chose a fatal chronic liver injury model, Fah-deficient induced CLF in mice. Transplanted adult HCs had successful liver regeneration, which remitted the liver injury resulting from the defect of tyrosine metabolism and kept most Fah-/- mice (4/5) survive at least 50 days from NTBC withdrawal. 


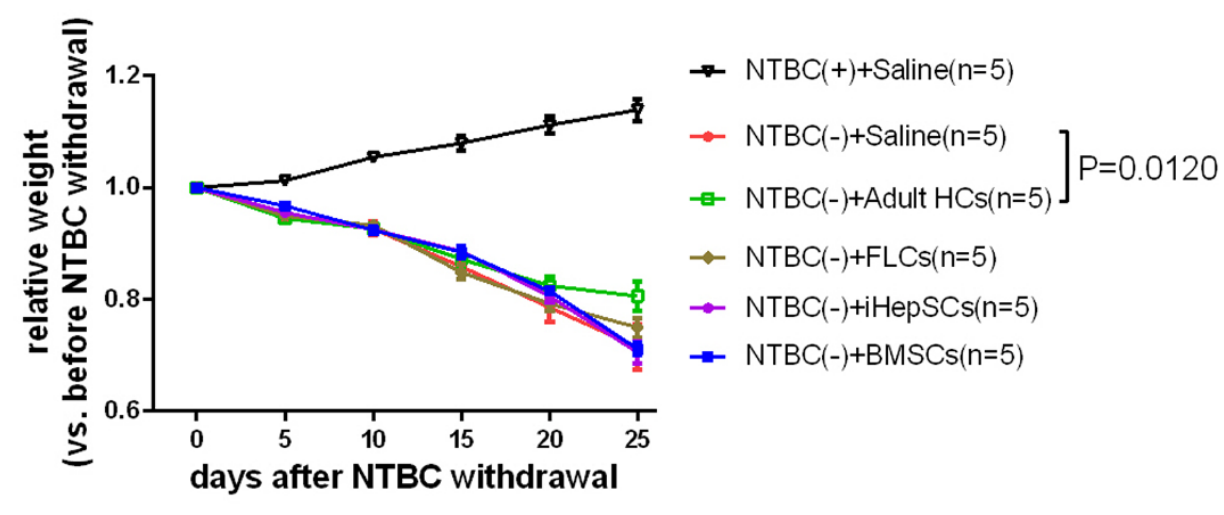

b

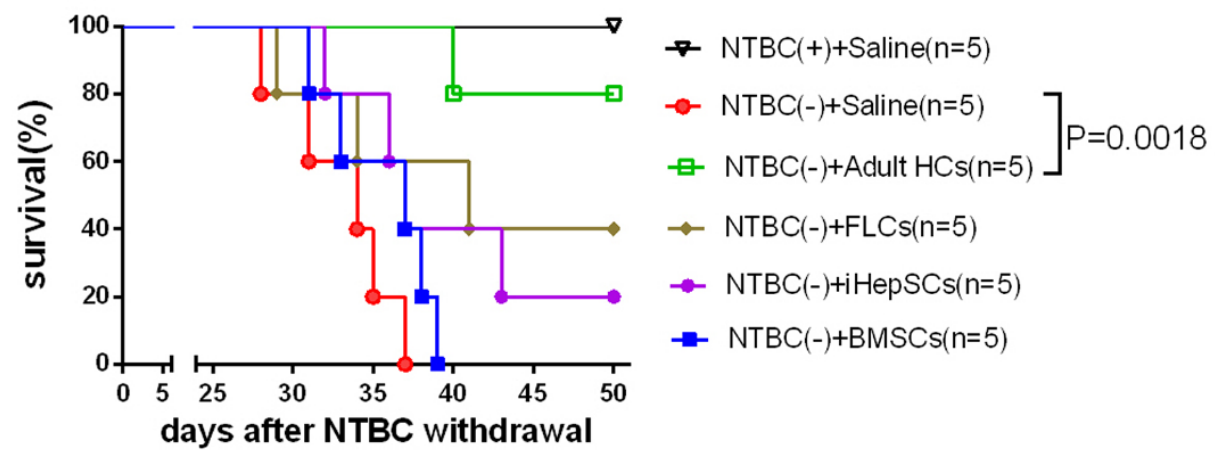

Figure 5 The body weights and survival times of Fah-/- mice with NTBC withdrawal and CBTs. (a) The relative body weight of the mice of the indicated groups after NTBC withdrawal (versus its own body weight before NTBC withdrawal). Data were mean with range ( $\mathrm{n}=5$ ). (b) Survival curves of the indicated groups. Log rank test showed the difference between the curves of NTBC(-) + Saline and NTBC(-) + adult HCs groups (n=5).

Although adult HCs belongs to terminal differentiation cells, it exhibits strong proliferating ability. In contrast, transplanted iHepSCs only showed weak liver regeneration 25 days after NTBC withdrawal, and just save one Fah-/- mice. Previous study showed the effective replantation of iHepSCs in Fah-/- mice without NTBC, but it need to more time, 8 weeks ${ }^{8}$. The body weight of Fah-/- mice had fallen by more than $30 \%$ on 25 days from NTBC withdrawal (Figure 5a). During this period of time, feeble vitality of mice and few replantation of iHepSCs may cause subtle differences between previous study $^{8}$ and our model. Transplanted FLCs also revealed weaker liver regeneration ability than adult HCs, and only rescue a small part of Fah-/- mice (2/5). Both Dhivya Haridass, et al. ${ }^{35}$ and Kamimura R, et al. $^{36}$ also reported that adult HCs had higher regeneration efficiency compared to FLCs and embryonic stem cells derived hepatic cells in their liver injury models. iHepSCs and FLCs need time and environment to completely develop into mature hepatocytes. This may be the cause resulting in that the efficiencies of liver regeneration of both iHepSCs and FLCs are lower than that of adult HCs. In the Fah-/- mice model, BMSCs still decreased expressions of TNF- $\alpha$ and IFN- $\gamma$ in the liver (Supplementary Fig. S2 online). Whereas inflammatory response is not the main cause of liver injury but an accompanied and relatively moderate change after NTBC withdrawal. Therefore, BMSCs transplantation had not deceased Fah-deficient induced liver injury. Meanwhile, the fast and effective transdifferentiation from MSCs to mature hepatocytes needs strict conditions. This may lead to rare Fah-positive cells in the Fah-/- mice with NTBC withdrawal and BMSCs transplantation.

In summary, ALF is a fast and severe liver damage, which results in dramatic disorder of liver function and often is accompanied by fierce inflammation, but CLF is a persistent and relatively mild liver injury. BMSCs and adult HCs are the optimal candidates of CBT for the fierce inflammation-associated ALF and metabolic disorderinduced CLF, respectively. The different characteristics of the diseases and the different specialities of CBTs make the different choice. This founding supports more clues for the reasonable choice of CBT for liver failure in the clinic.

\section{Methods}

Animal. C57BL/6 mice were obtained from the Shanghai Experimental Center, Chinese Science Academy, Shanghai. Fah-/- mice (129S4 x C57BL/6 background) were maintained with $7.5 \mathrm{mg} / \mathrm{L}$ NTBC in the drinking water in our laboratory. All animals were maintained at an animal facility under pathogen-free conditions and received humane care according to the animal protocols approved by the Shanghai Jiao Tong University Animal Care Committee (2013AE032102).

Isolation of adult HCs, FLCs and BMSCs from mice. All these three kinds of cells were obtained from C57BL/6 mice. Adult HCs were isolated from the liver of adult mice by a two-step collagenase perfusion method, which was described by Smit JJ, et $\mathrm{al}^{37}$. In brief, mice liver was perfused through the portal vein with D-Hank's solution (Invitrogen, Gland Island, NY) until blood free, and then with Hank's solution (Invitrogen) containing $0.5 \mathrm{~g} / \mathrm{L}$ collagenase IV (Sigma-Aldrich, St.Louis, $\mathrm{MO}$ ) for 20 minutes. The liver was scraped using a tweezers, and was filtered by a $100 \mathrm{~mm}$ strainer, and then was stirred in Hank's solution containing $2.5 \mathrm{~g} / \mathrm{L}$ pronase and $0.05 \mathrm{~g} / \mathrm{L}$ DNase (both from Sigma-Aldrich) for $20 \mathrm{~min}$ at $37^{\circ} \mathrm{C}$. Then the disaggregated material was filtered through a $70 \mu \mathrm{m}$ cell filter. After twice washing, cells were re-suspended in $45 \%$ Percoll (Sigma-Aldrich) and re-centrifuged at $50 \times \mathrm{g}$ for 10 minutes. After enrichment by isodensity purification in Percoll, cells were gently washed and centrifuged, and were re-suspended in saline solution.

FLCs were isolated and purified from the livers of mice embryos at day 13.5 by the method that was described by Haridass D, et $\mathrm{a}^{35}$. Briefly, fetal livers were removed under binocular microscope and were digested by collagenase/dispase (Roche, Mannheim, Germany) for 15 minutes at $37^{\circ} \mathrm{C}$. Then the cells were treated by red blood cell lysis buffer (Sigma-Aldrich) and washed twice in cold Dulbecco's modified Eagle's medium (DMEM, Invitrogen). Finally, the cell re-suspended in saline solution.

BMSCs were generated from bone marrow flushed out of the femur and tibia of mice (4-6 weeks old) as described previously ${ }^{30,38}$. In short, BMSCs were cultured in $\alpha$ minimal essential medium complemented with $10 \%$ fetal bovine serum (FBS), $2 \mathrm{mM}$ 
a
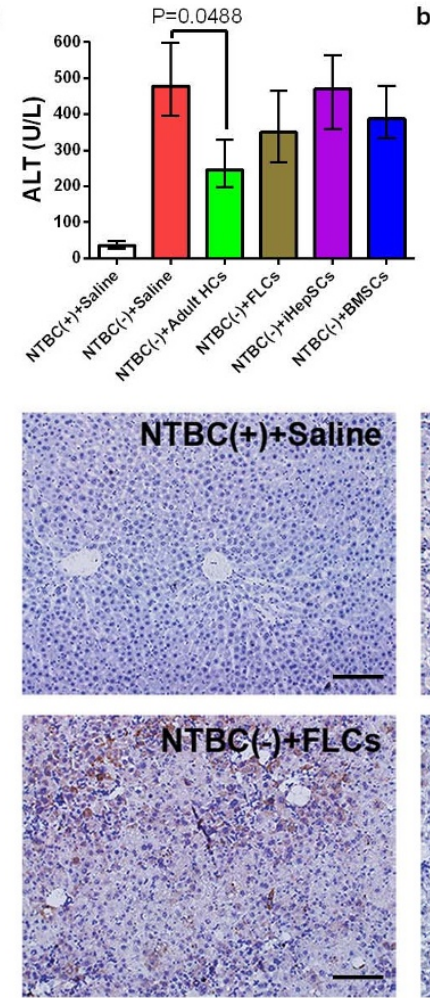

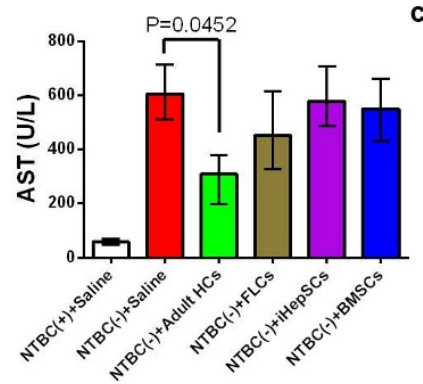

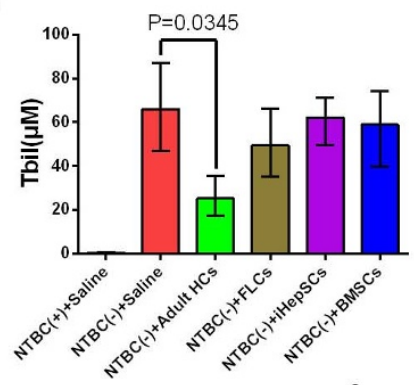

d

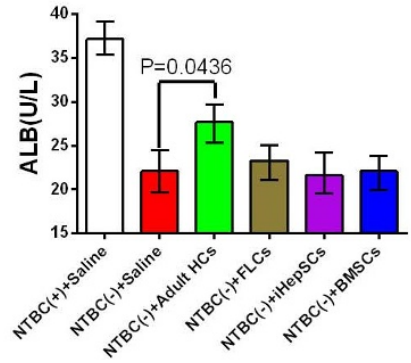

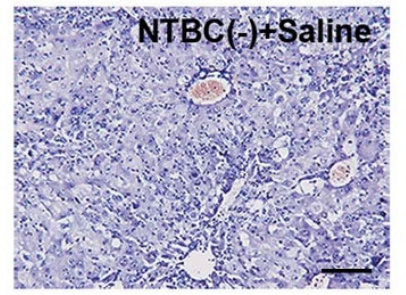

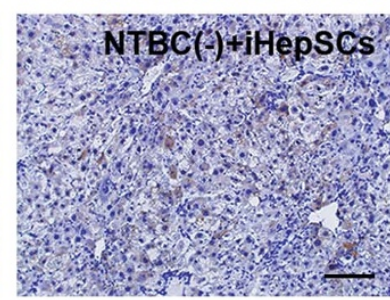

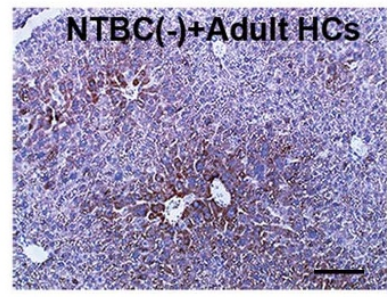

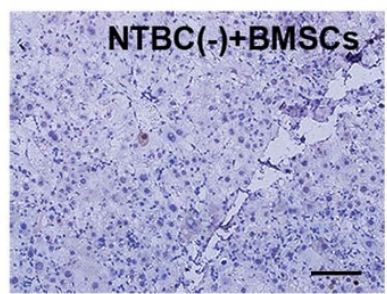

f

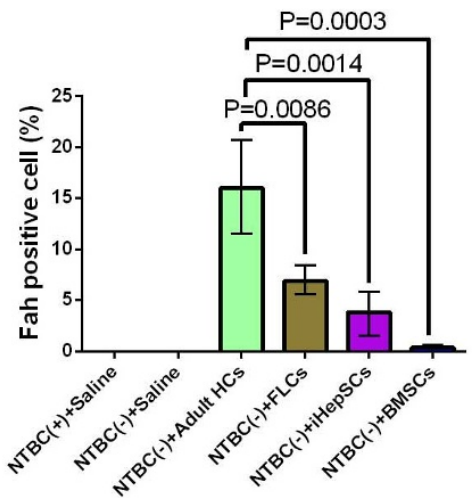

Figure 6 The levels of liver injury indicators and liver regeneration in the Fah-/- mice with NTBC withdrawal and CBTs. (a-e) The mice of the indicated groups were sacrificed 25 days after NTBC withdrawal. (a-d) The serum levels of ALT(a), AST(b), Tbil(c) and ALB(d) of mice from the indicated groups were detected. (e) Fah staining in liver sections of the indicated groups. Representative images were shown with an original magnification $\times 200$. Bar: $100 \mu \mathrm{m}$. (f) The ratios of Fah-positive cells were shown. Data were represented as mean with range $(\mathrm{n}=3)$.

glutamine and $1 \%$ penicillin/streptomycin (all from Invitrogen). Non-adherent cells were removed after 72 hours, and adherent cells were kept in media replenished every 3 days. Cells were utilized in the 5 th to 20 th passage.

Cell culture of iHepSCs. iHepSCs is a gift from Department of Cell Biology, Second Military Medical University, Shanghai, China, and were cultured as the description of Bing Yu, et al ${ }^{8}$. Briefly, iHepSCs were cultured in DMEM/F12 medium supplemented $10 \% \mathrm{FBS}, 1 \%$ penicillin/streptomycin, $0.1 \mathrm{mM} 2$-mercaptoethanol (Invitrogen), $10 \mathrm{ng} / \mathrm{ml} \mathrm{HGF}, 10 \mathrm{ng} / \mathrm{ml}$ EGF (both from R\&D Systems, Minneapolis, MN), $1 \times$ insulin-transferrin-selenium (ITS), $10^{-7} \mathrm{M}$ dexamethasone, $10 \mathrm{ng} / \mathrm{ml}$ nicotinamide, and $50 \mathrm{mg} / \mathrm{ml}$ gentamicin (all from Sigma-Aldrich).

The characterizations of adult HCs, FLCs, iHepSCs and BMSCs. Adult HCs, FLCs and iHepSCs were stained with rabbit anti-ALB (ab19196, 1:50), sheep anti-AFP (ab183147, 1:100) and rabbit anti-CK19 (ab52625, 1:200) (all from abcam, Cambridge, UK). Secondary antibodies included Alexa Fluor ${ }^{\circledR} 555$ Donkey AntiRabbit IgG (A21206) and Alexa Fluor ${ }^{\circledR} 488$ Donkey Anti-Sheep IgG (A11015) (both from Invitrogen, $1: 200)$.

BMSCs were analyzed by flow cytometry using anti-CD29-PE (102207), antiCD90-PE (205903), anti-CD105-PE (120407), anti-CD14-PE (123309), anti-CD34PE (128609) and anti-CD45-FITC (103104) (all from Biolegend, San Diego, CA). In brief, $1 \times 10^{5}$ cells were washed twice with PBS, re-suspended in $100 \mu \mathrm{l} \mathrm{PBS}$ containing specific antibody and incubated for $30 \mathrm{~min}$ at $4^{\circ} \mathrm{C}$. Then the cells were washed twice and re-suspended in $300 \mu \mathrm{l}$ PBS. Fluorescence analysis was performed with a flow cytometer (FACS Aria, BD).

BMSCs were treated with OriCell ${ }^{\mathrm{TM}}$ C57BL/6 Mouse Mesenchymal Stem Cell Adipogenic Differentiation Medium (Cyagen Biosciences Inc. Guangzhou, China) to induced adipogenic differentiation. Briefly, BMSCs were cultured in medium A (induction medium) for 3 days, and in medium B (maintenance medium) for 1 day, then were re-cultured in medium A. After 3 cycles, cells were cultured in medium B for 7 days. And then the cells were fixed in $4 \%$ formaldehyde, washed with PBS and stained with $0.2 \%$ oil-red O Solution (Sigma-Aldrich) to detect lipid droplets.

BMSCs were treated with OriCell ${ }^{\mathrm{TM}}$ C57BL/6 Mouse Mesenchymal Stem Cell Osteogenic Differentiation Medium (Cyagen Biosciences Inc.) to induced osteogenic differentiation for 3 weeks with medium changing every 3 days. And then the cells were fixed in $4 \%$ formaldehyde and washed with PBS, and were treated by von Kossa staining (Shanghai Showbio Biotech, Inc. Shanghai, China) to reveal calcium deposits.
CBTs for ALF and CLF in mice model. ConA (Sigma-Aldrich) was dissolved in saline solution $(5 \mathrm{mg} / \mathrm{ml})$. Then it was injected from tail vein $(25 \mathrm{mg} / \mathrm{kg})$ into male C57BL/6 mice (10-12 weeks old, weighing 25-27 g) to induce severe hepatitis and ALF. Adult HCs, FLCs, iHepSCs and BMSCs (all $10^{6}$ cells/100 $\mu \mathrm{l}$ saline solution) were intrasplenically injected into mice 2 hours after ConA injection, respectively.

The withdrawal of NTBC can induce CLF and ultimate death in the Fah -/- mice. While NTBC is withdrawn, adult HCs, FLCs, iHepSCs and BMSCs (all $10^{6}$ cells/ $100 \mu \mathrm{l}$ saline solution) were intrasplenically injected into the Fah $-/$ - mice (10-12 weeks old), respectively.

Biochemical analysis. The blood of mice was collected and serum was separated for biochemical analysis. Then the serum levels of ALT, AST, Tbil and ALB were determined using a FUJI DRI-CHEM7000 (Fuji Medical System, Tokyo, Japan) according to the manufacturer's instructions.

H\&E staining and Immunohistochemical staining. Liver sections of mice were preserved in $10 \%$ neutral-buffered formalin over night at $25^{\circ} \mathrm{C}$. Five random sections of paraffin-embedded liver tissues were stained with H\&E for histopathological analysis. For antigen retrieval, liver sections were performed by microwave-heating method in $0.01 \mathrm{M}$ citrate buffer ( $\mathrm{pH}$ 6). Then section were incubated in Fah antibody (Abbomax, San Jose, CA, 1:3000) over night at $4{ }^{\circ} \mathrm{C}$, followed by incubation with the HRP-conjugated anti-rabbit secondary antibody (ZSGB-BIO, Beijing, China) for 30 minutes at $37^{\circ} \mathrm{C}$. Then the slides were counterstained with Hematoxylin.

Isolation of lymphocytes and kupffer cells in mice liver. Intrahepatic lymphocytes and kupffer cells were isolated according to two method with slight modification ${ }^{39,40}$ Mice liver was treated with two-step collagenase perfusion, distributed and filtered like above methods. After filtering through $70 \mu \mathrm{m}$ cell strainer, cell suspensions were centrifuged at $50 \times \mathrm{g}$ for 10 minutes and the supernate was collected. The supernated was centrifuged at $250 \times \mathrm{g}$ for 3 minutes, and the cells were re-suspended in $30 \%$ Percoll. Intrahepatic lymphocytes and kupffer cells were isolated by centrifugation $(400 \times \mathrm{g})$ at room temperature for $15 \mathrm{~min}$ over a 30/70\% discontinuous Percoll.

Real-time quantitative polymerase chain reaction (qPCR). Liver sections of mice were soaked in RNALater (Qiagen GmbH, Hilden, Germany). And then the RNAs of total liver, intrahepatic lymphocytes and kupffer cells were extracted using Trizol Reagent (Invitrogen, Carlsbad, CA, USA), and were treated with RNase-free DNase (Promega, Madison, WI, USA) to deplete residual DNA. Complementary DNA was prepared by using oligo dT18-primers and MMLV reverse transcriptase (Promega). qPCR was performed using the LightCycler 480 system (Roche Diagnostics, 
Mannheim, Germany). The specific primers used to analyze gene expression: TNF- $\alpha$, Forward 5' -ATGAGCACAGAAAGCATGATC-3', Reverse 5' -TACAGGCTTGTCACTCGAA TT- $3^{\prime}$; IFN- $\gamma$, Forward $5^{\prime}$-GCCAAGACTGTGATTGCGGGGTTGTATCT-3', Reverse 5' -TAAAGCGC TGGCCCGGAGTGTAGACA-3'; FasL, Forward 5' -TCCACCTGCAGAAGGAACTG- $3^{\prime}$; Reverse 5' -GGTGTACTGGGGTTGGCTAT-3'; IL-10, Forward 5'-CCAAGCCTTATCGGAAATGA-3', Reverse $5^{\prime}$-TTTTCACAGGGGAGAAATCG-3'. Fold change in gene expression was determined by normalizing to endogenous $\beta$-actin: Forward 5 '-AGATGTGGATCAGCAAGCAG-3', Reverse 5' -GCG CAAGTTAGGTTTTGTCA-3'.

Statistical analysis. Data are presented as mean with range. Differences were analyzed by one-way ANOVA (Sidak correction). Survival curves were analyzed by log-rank test. A P-value of $<0.05$ was considered statistically significant. Statistical analyses were performed with GraphPad Prism 6.04 software (Graphpad Software, San Diego, CA, USA).

1. O'Grady, J. G., Alexander, G. J., Hayllar, K. M. \& Williams, R. Early indicators of prognosis in fulminant hepatic failure. Gastroenterology 97, 439-445 (1989).

2. Khashab, M., Tector, A. J. \& Kwo, P. Y. Epidemiology of acute liver failure. Curr Gastroenterol Rep 9, 66-73 (2007).

3. Wigg, A. J., McCormick, R., Wundke, R. \& Woodman, R. J. Efficacy of a chronic disease management model for patients with chronic liver failure. Clin Gastroenterol Hepatol Clin Gastroenterol Hepatol 11, 850-858 e851-854, doi:10.1016/j.cgh.2013.01.014 (2013).

4. Lee, W. M., Squires, R. H., Jr., Nyberg, S. L., Doo, E. \& Hoofnagle, J. H. Acute liver failure: Summary of a workshop. Hepatology 47, 1401-1415, doi:10.1002/ hep.22177 (2008).

5. Struecker, B., Raschzok, N. \& Sauer, I. M. Liver support strategies: cutting-edge technologies. Nat Rev Gastroenterol Hepatol, doi:10.1038/nrgastro.2013.204 (2013).

6. Dhawan, A., Puppi, J., Hughes, R. D. \& Mitry, R. R. Human hepatocyte transplantation: current experience and future challenges. Nat Rev Gastroenterol Hepatol 7, 288-298, doi:10.1038/nrgastro.2010.44 (2010).

7. Banas, A. et al. Rapid hepatic fate specification of adipose-derived stem cells and their therapeutic potential for liver failure. J Gastroenterol Hepatol 24, 70-77, doi:10.1111/j.1440-1746.2008.05496.x (2009).

8. Yu, B. et al. Reprogramming fibroblasts into bipotential hepatic stem cells by defined factors. Cell stem cell 13, 328-340, doi:10.1016/j.stem.2013.06.017 (2013).

9. Gantner, F., Leist, M., Lohse, A. W., Germann, P. G. \& Tiegs, G. Concanavalin Ainduced T-cell-mediated hepatic injury in mice: the role of tumor necrosis factor. Hepatology 21, 190-198 (1995)

10. Grompe, M. et al. Loss of fumarylacetoacetate hydrolase is responsible for the neonatal hepatic dysfunction phenotype of lethal albino mice. Genes Dev 7, 2298-2307 (1993).

11. Wang, X. et al. Cell fusion is the principal source of bone-marrow-derived hepatocytes. Nature 422, 897-901, doi:10.1038/nature01531 (2003).

12. Mizuhara, H. et al. Critical involvement of interferon gamma in the pathogenesis of T-cell activation-associated hepatitis and regulatory mechanisms of interleukin-6 for the manifestations of hepatitis. Hepatology 23, 1608-1615, doi:10.1053/jhep.1996.v23.pm0008675184 (1996).

13. Seino, K. et al. Contribution of Fas ligand to T cell-mediated hepatic injury in mice. Gastroenterology 113, 1315-1322 (1997).

14. Trautwein, C. et al. Concanavalin A-induced liver injury triggers hepatocyte proliferation. J Clin Invest 101, 1960-1969, doi:10.1172/JCI504 (1998).

15. Kaneko, Y. et al. Augmentation of Valpha14 NKT cell-mediated cytotoxicity by interleukin 4 in an autocrine mechanism resulting in the development of concanavalin A-induced hepatitis. J Exp Med 191, 105-114 (2000).

16. Louis, H. et al. Critical role of interleukin 5 and eosinophils in concanavalin Ainduced hepatitis in mice. Gastroenterology 122, 2001-2010 (2002).

17. Erhardt, A., Biburger, M., Papadopoulos, T. \& Tiegs, G. IL-10, regulatory T cells, and Kupffer cells mediate tolerance in concanavalin A-induced liver injury in mice. Hepatology 45, 475-485, doi:10.1002/hep.21498 (2007).

18. Matas, A. J. et al. Hepatocellular transplantation for metabolic deficiencies: decrease of plasms bilirubin in Gunn rats. Science 192, 892-894 (1976).

19. Grompe, M. Principles of therapeutic liver repopulation. Journal of inherited metabolic disease 29, 421-425, doi:10.1007/s10545-006-0311-2 (2006).

20. Huang, P. et al. Induction of functional hepatocyte-like cells from mouse fibroblasts by defined factors. Nature 475, 386-389, doi:10.1038/nature10116 (2011)

21. Huang, P. et al. Direct reprogramming of human fibroblasts to functional and expandable hepatocytes. Cell stem cell 14, 370-384, doi:10.1016/ j.stem.2014.01.003 (2014).

22. Yasui, O. et al. Isolation of oval cells from Long-Evans Cinnamon rats and their transformation into hepatocytes in vivo in the rat liver. Hepatology 25, 329-334, doi:10.1053/jhep.1997.v25.pm0009021943 (1997).

23. Wang, X. et al. The origin and liver repopulating capacity of murine oval cells. Proc Natl Acad Sci U S A 100 Suppl 1, 11881-11888, doi:10.1073/pnas.1734199100 (2003).

24. Oertel, M. et al. Purification of fetal liver stem/progenitor cells containing all the repopulation potential for normal adult rat liver. Gastroenterology 134, 823-832, doi:10.1053/j.gastro.2008.01.007 (2008)
25. Alison, M. R., Choong, C. \& Lim, S. Application of liver stem cells for cell therapy. Semin Cell Dev Biol 18, 819-826, doi:10.1016/j.semcdb.2007.09.016 (2007).

26. Aurich, I. et al. Functional integration of hepatocytes derived from human mesenchymal stem cells into mouse livers. Gut 56, 405-415, doi:10.1136/ gut.2005.090050 (2007).

27. Kuo, T. K. et al. Stem cell therapy for liver disease: parameters governing the success of using bone marrow mesenchymal stem cells. Gastroenterology 134 2111-2121, 2121 e2111-2113, doi:10.1053/j.gastro.2008.03.015 (2008).

28. Tuncer, C., Oo, Y. H., Murphy, N., Adams, D. H. \& Lalor, P. F. The regulation of Tcell recruitment to the human liver during acute liver failure. Liver Int 33, 852-863, doi:10.1111/liv.12182 (2013)

29. Cao, H. et al. Therapeutic potential of transplanted placental mesenchymal stem cells in treating Chinese miniature pigs with acute liver failure. BMC Med 10, 56, doi:10.1186/1741-7015-10-56 (2012).

30. Han, Z. et al. Immunosuppressive effect of bone marrow-derived mesenchymal stem cells in inflammatory microenvironment favours the growth of B16 melanoma cells. J Cell Mol Med 15, 2343-2352, doi:10.1111/j.1582 4934.2010.01215.x (2011)

31. Su, J. et al. Phylogenetic distinction of iNOS and IDO function in mesenchymal stem cell-mediated immunosuppression in mammalian species. Cell Death Differ 21, 388-396, doi:10.1038/cdd.2013.149 (2014).

32. Salomone, F., Barbagallo, I., Puzzo, L., Piazza, C. \& Li Volti, G. Efficacy of adipose tissue-mesenchymal stem cell transplantation in rats with acetaminophen liver injury. Stem Cell Res 11, 1037-1044, doi:10.1016/j.scr.2013.07.003 (2013).

33. Arkadopoulos, N., Papalois, A., Pataryas, T. H., Golematis, B. \& Papadimitriou, J. Experimental transplantation of hepatocytes in cases of toxic acute liver failure. An allograft model. Transpl Int 7 Suppl 1, S171-174 (1994).

34. Gupta, S., Rajvanshi, P., Irani, A. N., Palestro, C. J. \& Bhargava, K. K. Integration and proliferation of transplanted cells in hepatic parenchyma following Dgalactosamine-induced acute injury in F344 rats. J Pathol 190, 203-210, doi:10.1002/(SICI)1096-9896(200002)190:2203::AID-PATH521>3.0.CO;2-2 (2000).

35. Haridass, D. et al. Repopulation efficiencies of adult hepatocytes, fetal liver progenitor cells, and embryonic stem cell-derived hepatic cells in albuminpromoter-enhancer urokinase-type plasminogen activator mice. Am J Pathol 175, 1483-1492, doi:10.2353/ajpath.2009.090117 (2009).

36. Kamimura, R. et al. Comparative study of transplantation of hepatocytes at various differentiation stages into mice with lethal liver damage. Cell Transplant 21, 2351-2362, doi:10.3727/096368912X636957 (2012).

37. Smit, J. J. et al. Homozygous disruption of the murine mdr2 P-glycoprotein gene leads to a complete absence of phospholipid from bile and to liver disease. Cell 75, 451-462 (1993)

38. Liu, Y. et al. Effects of inflammatory factors on mesenchymal stem cells and their role in the promotion of tumor angiogenesis in colon cancer. J Biol Chem 286, 25007-25015, doi:10.1074/jbc.M110.213108 (2011).

39. Yan, J. et al. Parenchymal expression of CD40 exacerbates adenovirus-induced hepatitis in mice. Hepatology 53, 1455-1467, doi:10.1002/hep.24270 (2011)

40. Byun, J. S., Suh, Y. G., Yi, H. S., Lee, Y. S. \& Jeong, W. I. Activation of toll-like receptor 3 attenuates alcoholic liver injury by stimulating Kupffer cells and stellate cells to produce interleukin-10 in mice. J Hepatol 58, 342-349, doi:10.1016/ j.jhep.2012.09.016 (2013).

\section{Acknowledgments}

This project was supported by the Key Basic Research Project of China (Grant NO. 2011CB966203); Major State Scientific Research Program of China (Grant NO. 2012CBA01303); Key Project of National Natural Science Foundation of China (Grant NO 81030041); National Natural Science Foundation of China (Grant NO. 31171321, 81201584, 81372312, 81301715); Special Funds for National key Sci-Tech Sepcial Project of China (Grant NO.2012ZX10002-016, 2012ZX10002011-011); Shanghai Science and Technology Committee (Grant NO. 12431900802, 12ZR1454200); Innovation Program of Shanghai Municipal Education Commission(14YZ041)and Science Fund for Creative Research Groups, NSFC, China (Grant NO. 81221061). Thanks Yiping Hu, Zhiying He and Bing Yu (all work in the Department of Cell Biology, Second Military Medical University, Shanghai, China) for iHepSCs line.

\section{Author contributions}

K.S., X.Q.X. and J.X. contributed equally to this work. K.S., X.Q.X., X.W. and L.X.W conceived and designed the study. K.S., X.Q.X., S.F.J. and X.J.C. performed animal experiments. K.S. and J.X. performed biochemical analysis and liver section staining. X.J.C. and X.Z. performed molecular experiments. K.S., J.X. and X.Z. performed identification of cell characterizations. K.S., X.Q.X. and J.X. wrote the draft manuscript. L.X.W. finalized the manuscript. All authors read and approve the final manuscript.

\section{Additional information}

Supplementary information accompanies this paper at http://www.nature.com/ scientificreports

Competing financial interests: The authors declare no competing financial interests. 
How to cite this article: Sun, K. et al. Cell-based therapy for acute and chronic liver failures: Distinct diseases, different choices. Sci. Rep. 4, 6494; DOI:10.1038/srep06494 (2014).

(c) This work is licensed under a Creative Commons Attribution-NonCommercialBY NC SA ShareAlike 4.0 International License. The images or other third party material in this article are included in the article's Creative Commons license, unless indicated otherwise in the credit line; if the material is not included under the Creative Commons license, users will need to obtain permission from the license holder in order to reproduce the material. To view a copy of this license, visit http:// creativecommons.org/licenses/by-nc-sa/4.0/ 